\section{Nucleus Cuneatus}

John E. Mendoza

Department of Psychiatry and Neuroscience, Tulane Medical School and SE Louisiana Veterans Healthcare System, New Orleans, LA, USA

\section{Synonyms}

Cuneate nucleus

\section{Definition}

Nucleus located in the lower, dorsal portion of the medulla. It lies rostral to the pyramidal decussation and just lateral to the nucleus gracilis. This nucleus represents the rostral termination of the fasciculus cuneatus, the posterior column spinal pathway that carries information regarding proprioception, fine tactual discrimination (stereognosis) and vibratory sensations from the upper extremities and upper trunk. In turn, the nucleus gives rise to internal arcuate fibers which cross the midline of the medulla and, along with similar crossing fibers from the nucleus gracilis, form the medial lemniscus which terminates in the thalamus. Thus, a lesion in the lower and dorsal portion of the medulla affecting this nucleus can result in disturbances of proprioception, stereognosis and vibratory sense in the ipsilateral upper extremity.

\section{Cross-References}

- Fasciculus Cuneatus

- Medial Lemniscus 